\title{
A Map Representation of the ASET-RSET Concept*
}

\author{
Benjamin Schröder ${ }^{\mathrm{a}}$, Lukas Arnold ${ }^{\mathrm{b}, \mathrm{c}}$, Armin Seyfried $^{\mathrm{b}, \mathrm{d}}$ \\ ${ }^{a}$ Görtzen Stolbrink \& Partner mbB, Düsseldorf, Germany \\ ${ }^{b}$ Institute for Advanced Simulation, Forschungszentrum Jülich, Jülich, Germany \\ ${ }^{c}$ Computational Civil Engineering, Bergische Universität Wuppertal, Wuppertal, Germany \\ ${ }^{d}$ Computer Simulation for Fire Safety and Pedestrian Traffic, Bergische Universität \\ Wuppertal, Wuppertal, Germany
}

\begin{abstract}
Assessing life safety by comparing the available safe egress time (ASET) and the required safe egress time (RSET) is a prominent task in performance-based fire safety design. The calculation of a safety margin by subtracting RSET from ASET is a straightforward concept and is easy to understand. However, when the concept was developed, fire and evacuation models only provided punctual information derived from experimental correlations or hand calculations. Nowadays, complex computer models for fire and evacuation dynamics have become state of the art. However, the ASET-RSET concept has not adapted to these developments. While uncertainties related to the model input and the model application are widely recognised, uncertainties emerging from analysing the output only play a subordinate role.

Therefore, we introduce a map representation of ASET and RSET. The maps are generated by a spatial evaluation of the quantities used to determine ASET and RSET. Based on that, a difference map is introduced to represent the safety margin throughout the entire domain. Finally, a method is proposed to reduce the high information content of the difference maps to one scalar measure of consequences. This facilitates multivariate or risk-based analysis approaches and thus is able to reduce the uncertainties in performance-based design.

Keywords:

Performance-based Design, Life Safety Analysis, ASET, RSET, Safety Margin, Map Analysis, Consequence Quantification
\end{abstract}

\section{Introduction}

As alternative to the prescriptive fire safety design, the performance-based design approach has meanwhile established in many engineering standards,

${ }^{\star}$ This is a preprint version of the manuscript published in the Fire Safety Journal, which is referenced as DOI: 10.1016/j.firesaf.2020.103154.

Preprint submitted to Elsevier

July 17, 2020 
guidelines and handbooks worldwide [1, 2, 3. It can be utilised for assessing various protection goals and objectives related to fire safety. A prominent protection goal is life safety of building occupants in case of fire. According to [3] this goal can be specified by the objective that all occupants need to reach a safe place before they may be harmed by any effects of fire. Depending on the individual project characteristics, the assessment of this objective can be carried out in several ways.

One approach is to compare the available safe egress time (ASET) with the required safe egress time (RSET). While ASET is derived from the prediction of heat and smoke spread caused by a particular fire in a built environment, RSET is based on the time necessary for the occupants' movement towards a place of safety. The difference of both times is referred to as safety margin.

According to Babrauskas et al. 4, the root of the ASET-RSET concept was a smoke detector study conducted by NIST in the 1970s [5]. In this study, however, the terms RSET and ASET were not yet explicitly used. Instead, Babrauskas cites Cooper et al. [6] as the first article using the terminology ASET-RSET as well as the relationship ASET > RSET in conjunction with a particular margin of safety. Since Cooper's major focus was the estimation of ASET in different enclosure fires, he introduced RSET only on a conceptual level.

Over the years, a more refined view of the time intervals of both ASET and RSET has been condensed in the engineering timeline as described in [7. This timeline is meanwhile state of the art in fire safety engineering handbooks and standards worldwide [8, 9, 1, 10.

At the time when the ASET-RSET concept was developed, fire dynamics in enclosures were predominantly predicted by experimental correlations, which formed the basis for plume models such as 11,12 , 13. Later on, single- or multizone models [14, 15, 16] expanded the capabilities of fire safety engineering. All these models, however, have a macroscopic description of the system in common. This implies that the quantities of interest (e.g. time series of temperature, light extinction or gas concentrations) are solely evaluated at a few selected points in the built environment. The same applies to the calculation of egress times using hydraulic models such as [17, 18. These models also describe the egress process utilizing a macroscopic representation of pedestrian streams. Nowadays, models for the calculation of ASET and RSET provide a much higher level of complexity. For fire simulation, modern CFD-models [19, 20, 21] allow to resolve the spread of heat, smoke or other hazardous fire products with a much higher resolution in time and space. With regards to evacuation simulation, agent-based models 22, 23, 24, 25, 26 are increasingly used allowing to calculate the trajectories of individual pedestrians. The methodological frame for the application of the models has, however, not adapted to the advances mentioned above.

At a glance, ASET-RSET is a straightforward concept and easily comprehensible. However, considering the usage of modern engineering tools outlined above and claiming a well defined and verifiable procedure for the assessment of the safety of occupants, a closer look reveals several uncertainties and lacking definitions which could lead to false estimations regarding life safety. 
Uncertainties emerge from the input and the model level. It is impossible to consider all variations regarding occupancy, occupants, fire locations, fire developments, fuels and so forth. This challenge is addressed by considering a selection of scenarios and distributions of input quantities in conjunction with probabilities of particular scenario constellations. Furthermore, the models used to calculate the fire and egress dynamics will always be associated with limitations and simplifications. Having said that, uncertainties related to the output level often play a subordinate role. From a global perspective, RSET is normally related to the time when the last occupant left an enclosure or a building. In cases where performance-based design methods are applied, there is usually not one single location where a safety assessment is necessary. Especially when analysing complex built environments and a selection of different scenarios, the assessment is always based on a set of locations. Thus, in the understanding of the authors, ASET and RSET need to be understood as distributed values in space even in the frame of one single scenario.

As above indicated, the concept lacks specifications how and where to determine ASET and RSET. Usually, the performance criteria and the corresponding thresholds are controlled at selected locations in the building. However, the a priori definition of these locations as well as their comparison within a single scenario or set of scenarios is prone for incompleteness or misinterpretation. In concrete terms, it is not assured that the points selected to determine ASET are positioned at the location where the quantity of interest exceeds the threshold first. Taking into account a local perspective, it is not assured that the difference between ASET and RSET is larger than zero at every point in the building. The comparison of two times at only a few selected points in a large building does not address the spatial and temporal variations of the quantities of interest. In contrast, there is a particular demand to characterise the consequences of a particular scenario combination ideally by one scalar measure i.e. a particular safety margin.

This problem of a lacking consideration of spatial and temporal variations in ASET and RSET in large enclosures has already been identified by Poon [27. He proposes to extend the concept to an ASUT-RSUT concept (Available Safe Utility Time - Required Safe Utility Time). However, in his publication the implementation of the new concept is confined to a visual analysis of simulation snapshots.

An alternative approach to consider spatial and temporal variations adequately is the usage of the FED concept 28, 29. The application of this concept in the field of fire safety engineering has its origin in forensic analyses assessing residential fires in the United Kingdom. It is based on individual time/dose relationships for various fire effects resulting in impairment or prevention of an occupant's escape. In brief, this method is designated to evaluate potential exposures of occupants to multiple fire effects to arbitrary extent. In this respect, Purser rules out potential exposures caused by "sprinklered enclosures due to downdrag and loss of smoke buoyancy", or by the fact "that a system may be rendered inoperable". Finally, he also emphasises the appropriateness of "estimating the risk of injury and death throughout the life of the building [...] for 
a range of scenarios with varying probabilities" [28, p. 2310]. Because of these characteristics, the acknowledgement and adoption of the concept into building codes and engineering practice is heterogeneous. Last but not least, FED analyses require a close coupling of the applied fire and evacuation model in order to conjunct the agents' trajectories with the field data of the CFD simulations.

With these thoughts in mind, the focus of this paper is set on the extension of the ASET-RSET concept. In this case, there is no inherent need to couple the fire and evacuation models during the computation. Instead, the coupling is solely conducted when analysing the model output. This allows the users to apply independent fire and evacuation model frameworks. Nonetheless, the method can be applied to coupled calculations in the same way.

All in all, the paper addresses two major parts. Firstly, the ASET-RSET analysis itself is extended in a fashion that refrains from evaluating single performance criteria or monitoring selected locations in the computational domain. Instead, both ASET and RSET are rendered on maps. The subtraction of these maps yields the difference map, which equals the spatial calculation of the safety margin throughout the entire domain. This relieves the user from the challenges of localising the analyses and comparing their particular results. The method is presented in Section 2. Secondly, in the frame of a compact parameter study, the spatial representation of the safety margin is used to conduct different types of analysis, which are presented in Section 3 . The idea and reference implementation of the approach presented in this paper was published in [30. In this article, the methodology is extended by a mathematical formalisation and further considerations regarding map analysis. For that purpose, the methodology is introduced based on a simplified demonstration case. Finally, further considerations regarding limitations and a summary are stated in Sections 4 and 5.

\section{Map Generation}

\subsection{General idea}

Instead of evaluating time series of quantities relevant for life safety (e.g. light extinction, temperature or evacuated occupants) only at a few selected locations, the full information content of state-of-the-art models for both fire and evacuation simulation will be utilised.

The methodology will be introduced based on a deliberately simple demonstration case. The individual steps of the approach are formally described in detail in the following subsections. Although this demonstration case could be assessed with a traditional ASET-RSET analysis or even by consideration, it provides a straightforward setup for understanding the methodology and highlights its benefits and shortcomings.

A more complex example is provided in [30]. In this work, the abovementioned methods were developed in order to conduct multivariate life safety analyses in an existing underground station. The station has in total three levels and a gross floor area of $7000 \mathrm{~m}^{2}$. The study covers a deterministic, multivariate 
analysis of approximately 100 fire scenarios in combination with approximately 80 evacuation scenarios. The results show highly variable fire dynamics due to multiple fire locations, design fires and the complex built environment. In conjunction with that, the pedestrian dynamics are affected by different population sizes, pre-movement times and route choice assumptions. The proposed map analysis was conducted for over 8000 scenario combinations and the scalar measure of consequences was utilised for clustering techniques.

In this article, however, the simplified demonstration case is set up the following way, see Figure 1(a) for an outline of the geometry:

- a single compartment, $10 \mathrm{~m} \times 30 \mathrm{~m} \times 3 \mathrm{~m}$ (floor area $\times$ height),

- no openings, but a door, which is the exit for the evacuation,

- a fire located in the opposite corner to the exit, as the fire properties are of no importance for this work, we deliberately leave out details,

- the room is occupied by 100 pedestrians by default and

- the pedestrians' movement towards the exit starts instantaneously.

The first step is the choice of a set of criteria for the ASET and RSET determination. For this purpose, the relevant performance criteria are conjunct with particular thresholds.

In contrast do the classical approach, these criteria will be evaluated at multiple locations. Therefore, a discretisation of the domain of interest is needed. Here, a spatial discretisation, in form of a map, is defined. In the following, a map is a set of points, i.e. map elements, that represent (parts of) the ground plan of the compartment, see Figure 11(b). These points may either represent disjoint areas, where their total union is the ground plan, or the points' neighbourhoods. In both cases the local ASET-RSET criteria are evaluated in the corresponding areas.

As these criteria are based on temporal data like light extinction or the position of agents, they are evaluated at each point of a map for each stored data set during the computation. For every map element, the resulting maps are combined to the minimal values of the local ASET and to the maximal values of the local RSET. Finally, the ASET and RSET maps are related to each other by creating a difference map, which allows to identify locations, where e.g. the local RSET values are higher than the local ASET values.

Although the resulting difference map allows a detailed spatial analysis, a direct comparison, i.e. a ranking, of scenarios is not readily possible. For that purpose, Section 3 provides different approaches to derive single scalar measures from the difference maps. As within the classical understanding of ASET-RSET analyses, this renders quantitative comparison of multiple scenarios possible.

The following sections provide a detailed definition of the ASET maps (Section 2.2), the RSET maps (Section 2.3) as well as the difference maps (Section 2.4). The simulation input files, the simulation output data as well as a basic implementation of the presented analysis methods are publicly available 31]. 


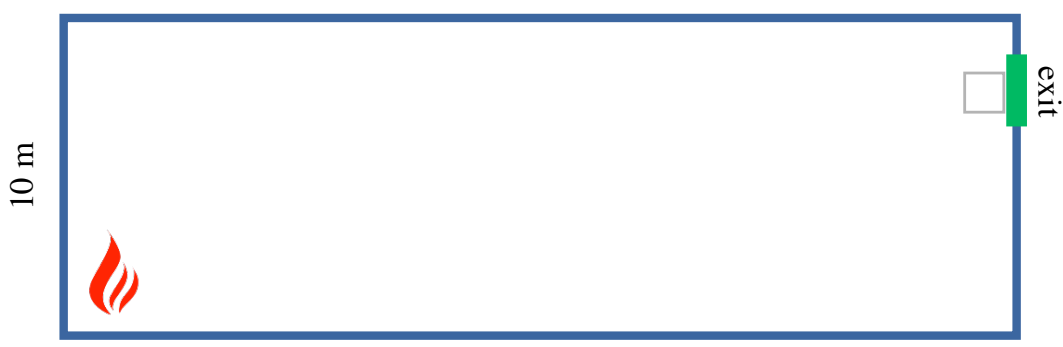

$30 \mathrm{~m}$

(a)

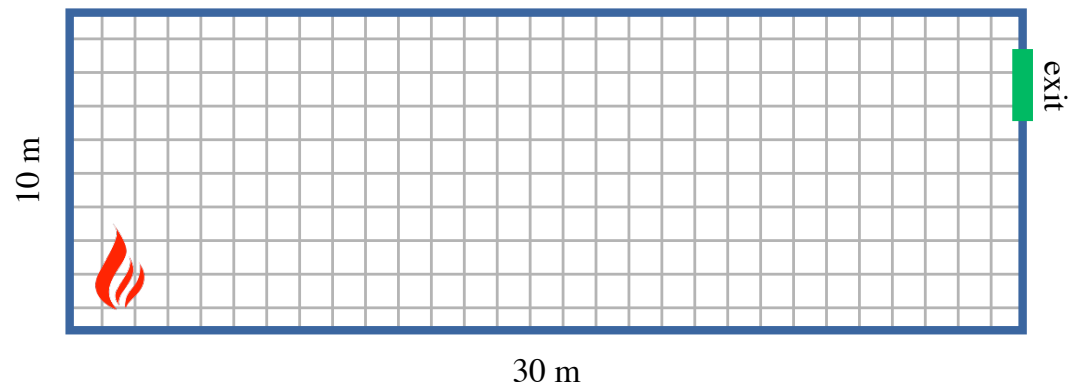

(b)

Figure 1: Setup of the demonstration case, including a single exit for evacuation and a fire location. The top figure (a) indicates the classical approach of ASET-RSET analysis where one selected point of interest, indicated by the grey square, is used. The lower figure (b) sketches the data structure representing measurements of the quantities of interest over the whole area. A grid is introduced to decompose the room into measurement areas. This data structure allows for the generation of ASET and RSET maps.

\subsection{ASET maps}

\subsubsection{Performance Criteria}

In the following it is assumed, that performance criteria are based on physical quantities $q=q(x, y, z, t)$ like light extinction, air temperature or gas concentrations of $\mathrm{CO}, \mathrm{O}_{2}$ or other effluents. Thus, an ASET criterion $K_{i}$ for a quantity $q_{i}$ can be expressed as a simple relation to a threshold $q_{i, t h}$. Depending on the physical quantity, this may result in one of the following inequalities

$$
K_{i} \equiv q_{i} \geq q_{i, t h} \quad \text { or } \quad K_{i} \equiv q_{i} \leq q_{i, t h} .
$$

The above inequalities are presented for a single quantity, but are applicable to the full scalar field leading to $K_{i}=K_{i}(x, y, z, t)$.

In general, these relations can be more complex, but shall be defined as true, if the quantity value reached a critical region. 


\subsubsection{Discretisation}

As stated above, the evaluation of ASET at a given map element $M$ at $\left(x_{m}, y_{m}\right)$ is based on local quantities. These quantities are four-dimensional scalar fields, i.e. created by a computational fluid dynamics (CFD) model. As we assume, that this data is the result of a numerical simulation, the spatial and temporal dimensions are discretised and represented by data points. Now each map element is assigned a set $X_{m}$ of all data points which are located at a given elevation above the compartment's floor. The choice of map elements is not bound to the numerical mesh of the simulation model. Thus, if the extension of a map element is larger than the size of the numerical mesh, it will contain the accordingly greater number of points in $X_{m}$ compared to other map elements. In general, this may lead to an uneven distribution of the simulation data points but allows a flexible choice of the analysis domain.

\subsubsection{Creation}

The final goal is to find the first point in time at each map element, when any of the monitored quantities has reached a critical value.

With the above definitions, a set of time points $T_{m, i}$, when the threshold of a performance criterion $i$ is reached or exceeded at any data point of the set $X_{m}$, is given as

$$
T_{m, i}=\left\{t \mid K_{i}(\vec{x}, t) \forall \vec{x} \in X_{m}\right\} .
$$

The union of all $T_{m, i}$, i.e. all performance criteria at a map element, contains all points in time when any of the quantities have reached critical values. Therefore, the first time point $t_{m}$ at which any criterion was met in the data set $X_{m}$ assigned to a map element $M$ is

$$
\operatorname{ASET}_{m}=\operatorname{ASET}\left(x_{m}, y_{m}\right) \equiv t_{m}=\min \left(\bigcup_{i} T_{m, i}\right)
$$

This procedure for a single map element is used to construct all map elements $\mathrm{ASET}_{m}$ and their combination leads to the multi-criteria ASET map.

\subsubsection{Example}

With the previous definitions, the large data amount of of CFD simulations is utilised to render ASET on a map. In the demonstration case, the only performance criterion is the light extinction coefficient. In accordance with [32, the corresponding threshold is chosen as $0.23 \mathrm{~m}^{-1}$. In order to roughly represent a human's height, the data evaluation is based on the data slices at an elevation of $h_{0}=2 \mathrm{~m}$ above floor level. Therefore, the map elements' data sets $X_{m}$ are restricted to data points at a fixed height $h_{0}$.

In the next step, the map's spatial resolution needs to be specified. For this purpose, the spatial discretisation of the fire simulation domain can be adopted. However, the required resolutions for accurate CFD simulations are usually higher than necessary for ASET analyses. Therefore, it is arguable to coarsen 
the resolution. In this paper, the spatial discretisation of the CFD domain is $\Delta x=0.2 \mathrm{~m}$. Comparative studies showed that an ASET map resolution of $0.6 \mathrm{~m}$ is sufficient for engineering purposes.

In the next step, the temporal resolution of the analyses is addressed. Depending on the specified output frequency, CFD simulations can provide enormous amounts of output data. However, for engineering questions and the underlying uncertainties, time scales of half-minute intervals should be sufficient for assessing life safety. This is motivated by the fact that fire and pedestrian dynamics in built environments can be adequately described with this granularity of the output data. Note that this is not equivalent to the required temporal discretisation of numerical models. For the demonstration case, a time interval of $\Delta t=10 \mathrm{~s}$ is chosen because of its spatial and temporal compactness.

In the last initialisation step, the ASET map is entirely filled with the maximum ASET as shown in the first row of figure 2. In the demonstration case, the maximum ASET is chosen as $t_{\text {end }}=120 \mathrm{~s}$.

According to the routine formally introduced above, the ASET map generation is an iterative process starting from $t_{\text {start }}=0 \mathrm{~s}$ and terminating at $t_{\text {end }}=120 \mathrm{~s}$. Four intermediate time steps of this analysis are shown in Figure 2. In the demonstration case, it turns out that the intermediate ASET maps remain unchanged from row 3 to row 4 . This implies that the threshold of $0.23 \mathrm{~m}^{-1}$ is exceeded in every map element after at least 80 seconds. The final output of the analysis is one single ASET map as shown in the last row of Figure 2. It illustrates the spatial distribution of the available safe egress times and can be understood as the fingerprint of the underlying fire scenario. 

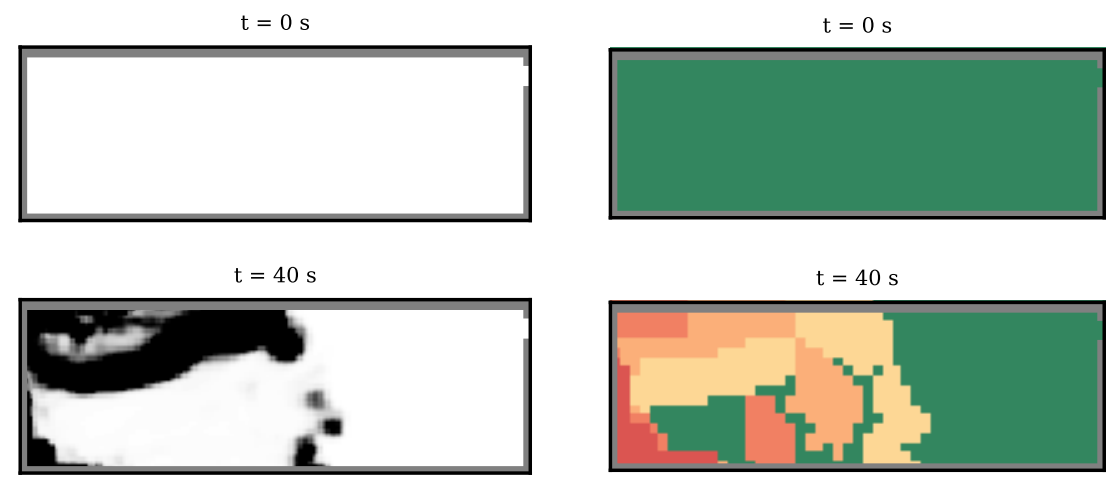

$\mathrm{t}=80 \mathrm{~s}$

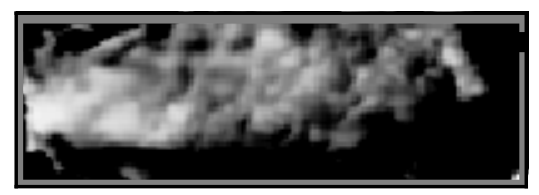

$\mathrm{t}=80 \mathrm{~s}$

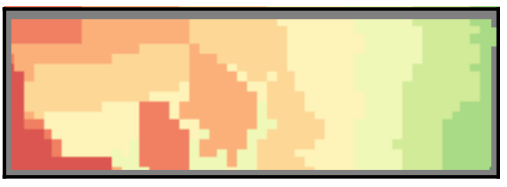

$\mathrm{t}=120 \mathrm{~s}$

$\mathrm{t}=120 \mathrm{~s}$
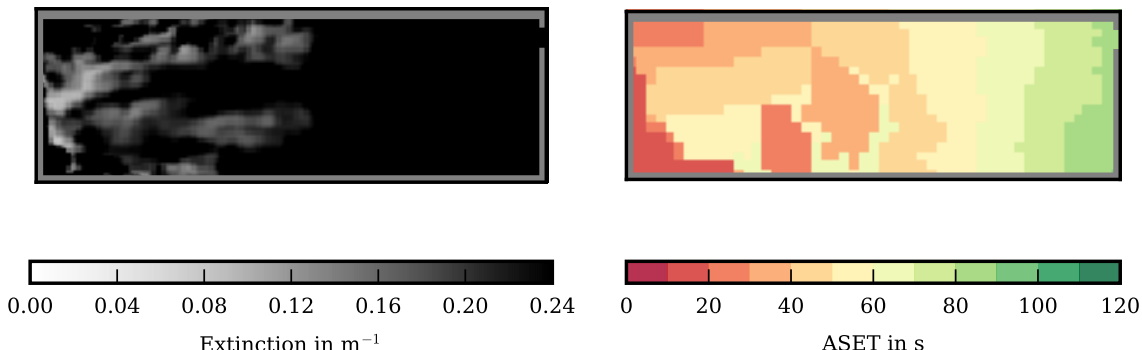

Figure 2: Process of ASET map generation in time. The left column shows evolution of the light extinction coefficient due to a fire starting in the bottom left corner of the room. The extinction is visualised as two-dimensional time series (slice) $2.00 \mathrm{~m}$ above the floor. Based on a threshold of $0.23 \mathrm{~m}^{-1}$, the right column illustrates the development of the corresponding ASET map.

Since the resulting ASET map refers to only one performance criterion, i.e. extinction, the entire process can be repeated for arbitrary performance criteria. This would lead to the last step, which would cover the elementwise computation of the minimum of all ASET maps per criterion. The final result, i.e. the multi-criteria ASET map, which also covers the gas temperature (threshold at $45^{\circ} \mathrm{C}$ ) is illustrated in Figure 4 (top) later-on.

\subsection{RSET maps}

\subsubsection{Creation}

For the sake of simplicity, the creation of the RSET maps assumes a similar floor decomposition as outlined in Section 2.2.2. Different map definitions are 
possible, while the operations on them have to be adequately addressed.

The basic idea of the analysis is to evaluate every single agent's evacuation path, i.e. the $i$-th trajectory $p_{i}(t)=p_{i}(x, y, t)$. The exclusion of the $z$ dimension requires the definition of an elevation $h_{0}$, which is used to assess the acceptance criteria. This allows the reduction of the data to be processed to only the investigated level of the building. In order to assign parts of the trajectory to a map element at $\left(x_{m}, y_{m}\right)$, the distance between both is computed. If cubic map elements with a width of $w$ are assumed, this results in the following set of time points $T_{m, i}$ at which the trajectory was within a distance $w / 2$ to the centre of the map element

$$
T_{m, i}=\left\{t \mid\left\|p_{i}(t)-\left(x_{m}, y_{m}\right)\right\|_{\infty}<\frac{w}{2}\right\} .
$$

It should be noted, that here the maximum norm is used to represent the cubic shape of an element. This formula can be generalised to any appropriate distance norm. In the similar fashion as in Equation 3, the value of the RSET map element $\mathrm{RSET}_{m}$ is given by the maximal time of the union of all trajectories passing the element.

$$
\operatorname{RSET}_{m}=\operatorname{RSET}\left(x_{m}, y_{m}\right) \equiv t_{m}=\max \left(\bigcup_{i} T_{m, i}\right)
$$

In this step, it is also possible to directly incorporate multiple repetitions of an evacuation simulation by compiling all trajectories into a single set.

\subsubsection{Example}

The initialisation of the RSET map is similar to the routine described in Section 2.2. However, some additional considerations are required for the spatial and temporal discretisation of the analysis.

In the first instance, the spatial discretisation needs to represent or at least be able to capture characteristic features of the built environment, e.g. narrow exits, aisles or stairs adequately. Also here, comparative studies showed that an RSET map resolution of $0.6 \mathrm{~m}$ is sufficient for engineering purposes.

With regards to the temporal resolution, the analysis has to comply with the condition that no information on the movement of a single agent is lost. Therefore, the time step $\Delta t$ should be chosen in a way that the maximum walking distance of an agent in this time is not larger than the width $w$ of a map element. Given a maximum velocity $v_{\max }$ the time discretisation $\Delta t$ is given by

$$
\Delta t \leq \frac{w}{v_{\max }} .
$$

With the default values of $w=0.6 \mathrm{~m}$ and $v_{\max }=1.2 \mathrm{~m} \mathrm{~s}^{-1}$ this results in a temporal discretisation smaller than $0.5 \mathrm{~s}$. In the last preparative step, the RSET map is initialised with non-valid number values in order to represent elements of the RSET map which have not been occupied yet. 
To update the RSET map based on the calculated pedestrian dynamics, the simulated agents' trajectories are analysed. For that purpose, the trajectories need to comprise at least the following information: time, agent ID, x-position, y-position, and z-position. If RSET maps are required for multiple floors, the zposition is required to filter the trajectories accordingly. The trajectories can be structured in multiple ways, e.g. time-wise or agent-wise and may be handled with different file formats, e.g. csv, txt, or xml. In the frame of this work, the trajectories are structured time-wise using an xml-schema. This allows for convenient data processing techniques, which is preferable since trajectories may obtain remarkable file sizes.

Figure 3 provides an overview of the analysis with four exemplary intermediate states of the RSET map. The final RSET map can be found in the last row. It is representing not an individual but a space-related interpretation of the required safe egress time. 

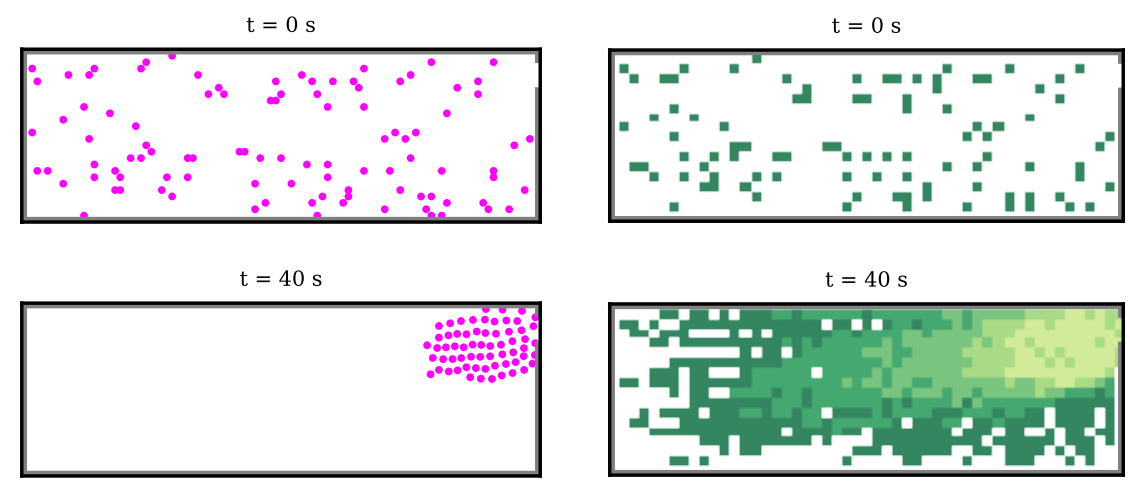

$\mathrm{t}=80 \mathrm{~s}$

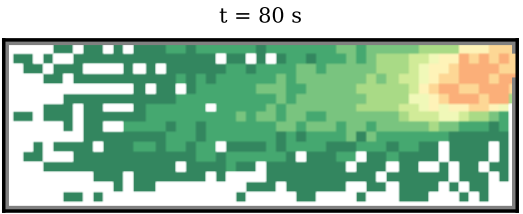

$\mathrm{t}=120 \mathrm{~s}$
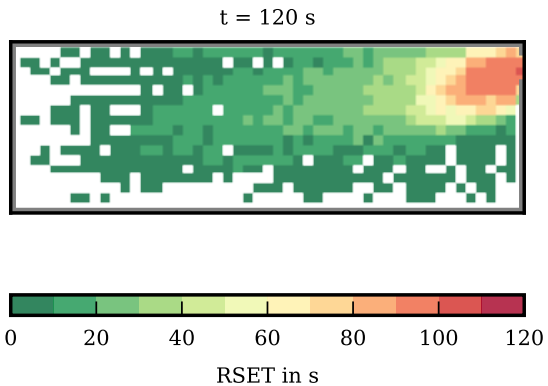

Figure 3: Process of RSET map generation. The left column shows the pedestrian's positions derived from the trajectories. The right column illustrates the development of the corresponding RSET map.

So far, the RSET map only represents one realisation of a particular evacuation scenario. With regards to the randomised initialisation of microscopic evacuation simulations, it is preferable to incorporate multiple realisations into the analysis.

For the demonstration case, a total number of ten realisations has been used to derive the RSET map as finally shown in Figure 4 (middle). Here, the global maximum of all underlying RSET maps (per realisation) has been computed. However, it is also possible to conduct further statistical processing, e.g. the calculation of percentiles regarding RSET. Furthermore, it is a promising approach to utilize a set of RSET maps (multiple realisations) to conduct a convergence analysis as proposed in [33]. Further reading on that topic can be found in 30. 


\subsection{Difference maps}

After the completion of generating maps for both ASET and RSET, it is possible to calculate the difference map DIFF of both quantities. If the map elements were chosen equally for both maps, this is conducted by subtracting RSET from ASET element-wise as shown in Equation 7

$$
\mathrm{DIFF}_{m}=\mathrm{ASET}_{m}-\mathrm{RSET}_{m}
$$

This map represents the spatial distribution of the so-called safety margin. As long as the difference is greater or equal zero, the ASET-RSET constraint is satisfied. With regards to the demonstration case, the underlying ASET and RSET maps as well as the corresponding difference map are illustrated in Figure 4 (bottom). 

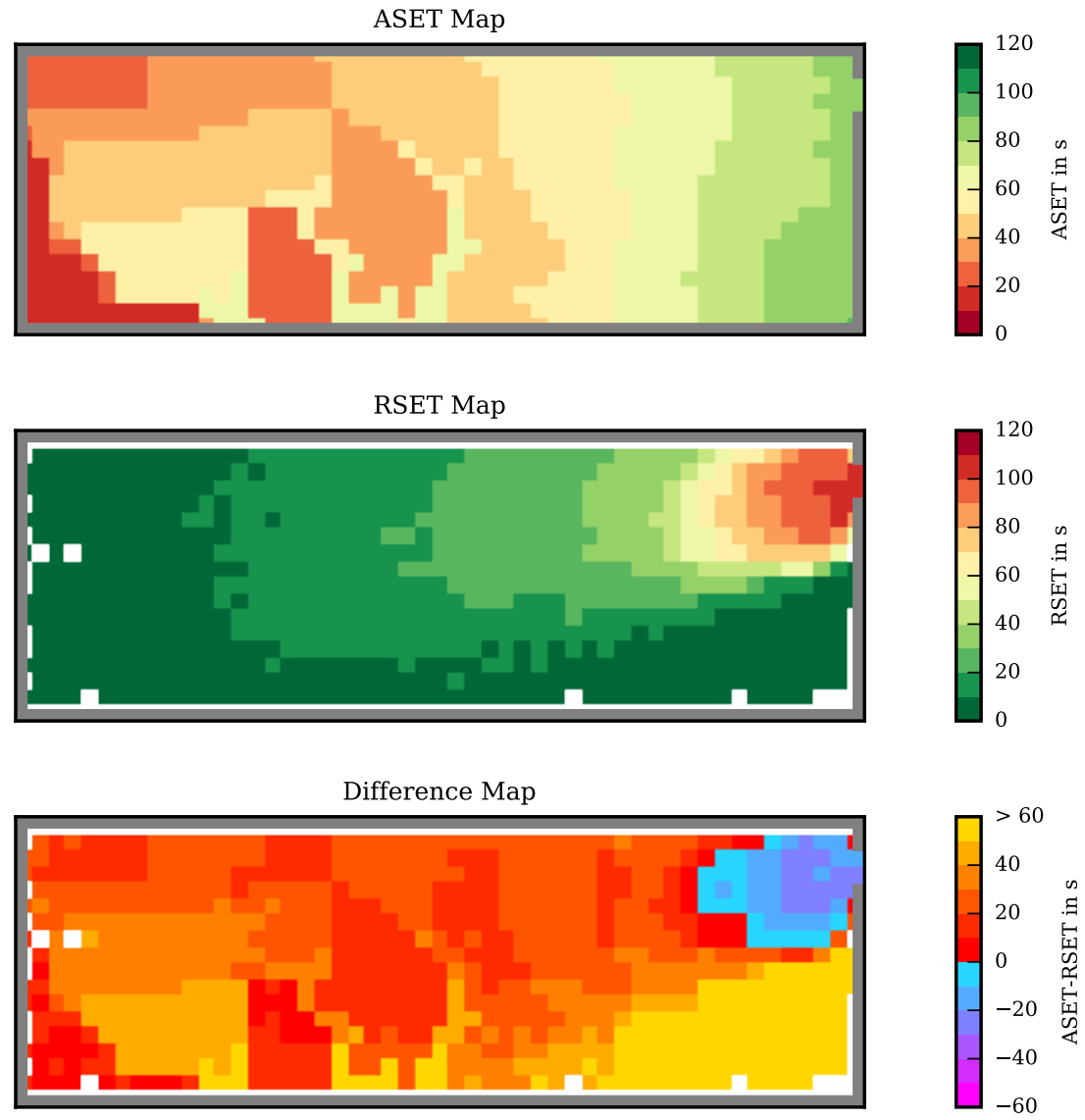

Figure 4: Top: ASET map derived from the light extinction coefficient and temperature. Despite the small compartment size, ASET spreads remarkably from almost 0 to 80 seconds. Centre: RSET map incorporating multiple numerical realisations. White elements show areas that were not traversed by any pedestrian despite multiple realisations. Bottom: Difference map representing the spatial distribution of ASET minus RSET. Here, the system is close to the limiting state of ASET - RSET $=0$ in proximity to the seat of fire and towards the exit. Thus, the safety margin is locally less than zero.

One of the main features of the difference map is the visualisation of the area(s) where the ASET-RSET condition is violated. Areas with negative values represent places where the evacuees were exposed to unacceptable fire conditions. The value indicates how long this critical state lasts. However, we note that the exposure time of individuals cannot be concluded from this value. This value allows the identification of the critical locations during the evacuation process as well as an evaluation of the duration of these critical states. Further analyses on the basis of difference maps are highlighted in the following section based on a simple parameter study. 


\section{Map Analysis}

The spatial representation of the safety margin in the difference map should already represent an advancement for data visualisation in engineering practice. However, especially when analysing scenario ensembles or when conducting risk analyses, there is a particular need to characterise a scenario combination by a single number. In this section, different approaches for analysing the difference maps are discussed.

The map analysis is conducted based on a simple parameter study using the following variations of the fire and evacuation scenarios:

- maximum heat release rate $\mathrm{HRR}_{\max }: 30 \mathrm{~kW}, 60 \mathrm{~kW}, 90 \mathrm{~kW}$ and $120 \mathrm{~kW}$,

- number of occupants $N: 25,50,75$ and 100 .

This section shows how the difference map can be used to estimate the consequences of a particular scenario combination.

As discussed at the end of the last section, negative values in the difference maps indicate areas with critical states. These critical states have a temporal and spatial dimension. Both dimensions may be separately used to estimate the consequences. In addition, a combined analyses using a measure in the dimension of space-time is proposed.

\subsection{Temporal and spatial extent of consequences}

At a glance, the difference map indicates if and to what temporal extend the limiting state has been exceeded. This information is provided by the minimum time in the difference map, i.e. the safety margin. This approach is equivalent to the classical interpretation of the ASET-RSET concept.

Let's carry out the following experiment in mind: We double the occupancy of the demonstration case and we add a second exit. Although many different aspects affect the decision making process, the route choice towards both exits is assumed based on the shortest path. It turns out that the temporal extent, i.e. the safety margin, remains more or less unchanged. A comparison of both scenarios based on the safety margin would yield the conclusion that the consequences are similar, although the modified demonstration case comprises two times more occupants who may exceed the limiting state. Thus, the consequences associated with the modified demonstration case should be higher.

The reason for this is that the temporal measure lacks any information about the spatial extent of the area where the safety margin is negative. Therefore, another measure of consequences is the overall area where the limiting state has been exceeded. Based on the map's spatial discretisation every map element represents a floor area. In the demonstration case, the map element area is $(0.6 \mathrm{~m})^{2}=0.36 \mathrm{~m}^{2}$. Hence, multiplying this area by the number of map elements with a difference value of less than zero yields the overall area where the limiting state has been exceeded. In analogy to the first measure, there is no information included about the duration of the violation. 
With regards to the input variations introduced in this section, the response of the above-mentioned measures is illustrated in Figure 5.
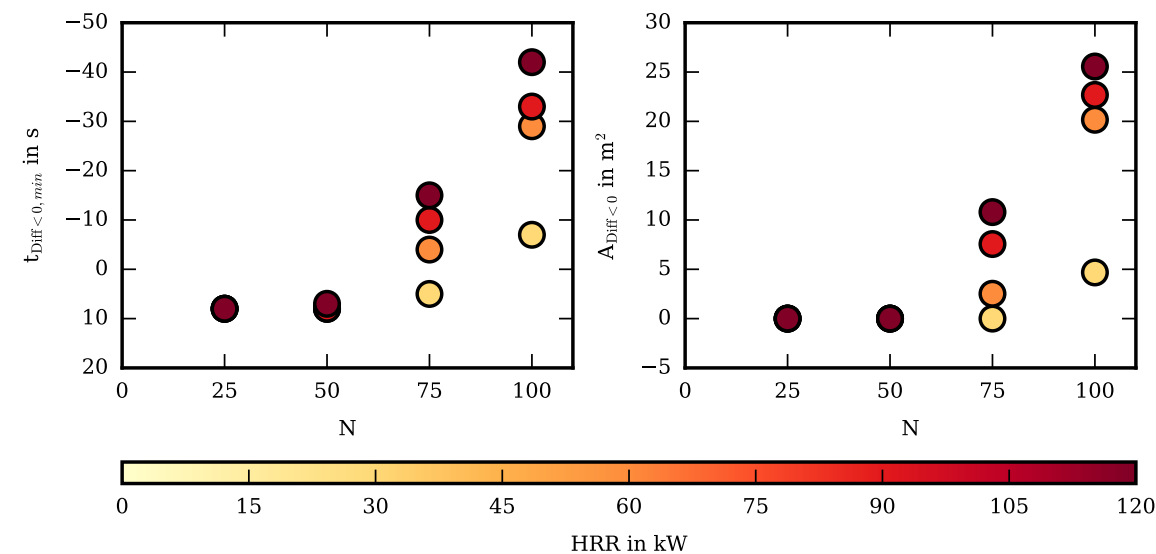

Figure 5: Scatter plots illustrating the response of minimum difference of ASET and RSET (left) and the corresponding area where the limiting state is exceeded (right). Input variations are four different heat release rates and four varying numbers of occupants. The response of both measures reveals similar patterns.

The analysis shows the obvious result that ASET-RSET is increasingly violated at a higher number of occupants in conjunction with higher heat release rates. In this respect, both time and area reveal similar responses to the parameter variations.

\subsection{Spatio-temporal extent of consequences}

So far, the two introduced measures of consequences either account for the temporal or the spatial exceedance of the limiting state. However, with regards to comparing or ranking a set of scenario combinations, it may be inadequate to solely make use of these two measures. In anticipation of Figure 7 , the difference maps can be transformed to histograms, whose negative fraction is of most interest.

On the basis of two hypothetical scenarios A and B, Figure 6 shows similar characteristics in terms of the temporal and the spatial exceedance of the limiting state. 

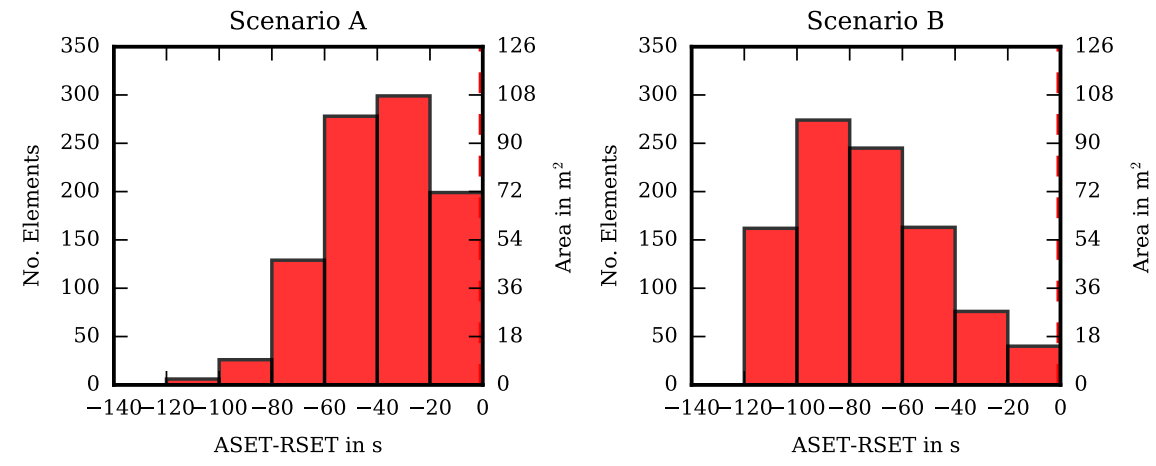

Figure 6: Difference map histograms of two hypothetical scenarios A and B. Both scenarios would yield the same time difference i.e. safety margin and area where the limiting state was exceeded. However scenario B includes more elements with larger ASET-RSET differences.

Nonetheless, the shape of the histograms is apparently different since scenario B yields a higher number of observations more distant from the limiting state than scenario A. Such characteristics can especially be induced by considering additional complexity on the input level, e.g. time-dependent design fire curves or pre-movement time distributions.

Having said that, the third measure of consequences conjuncts the spatial and temporal extend to one scalar measure. For that purpose, a histogram representing the distribution of ASET-RSET values in the difference map is computed. Figure 7 shows the histogram derived from the difference map of the demonstration case (see Figure 4 ).
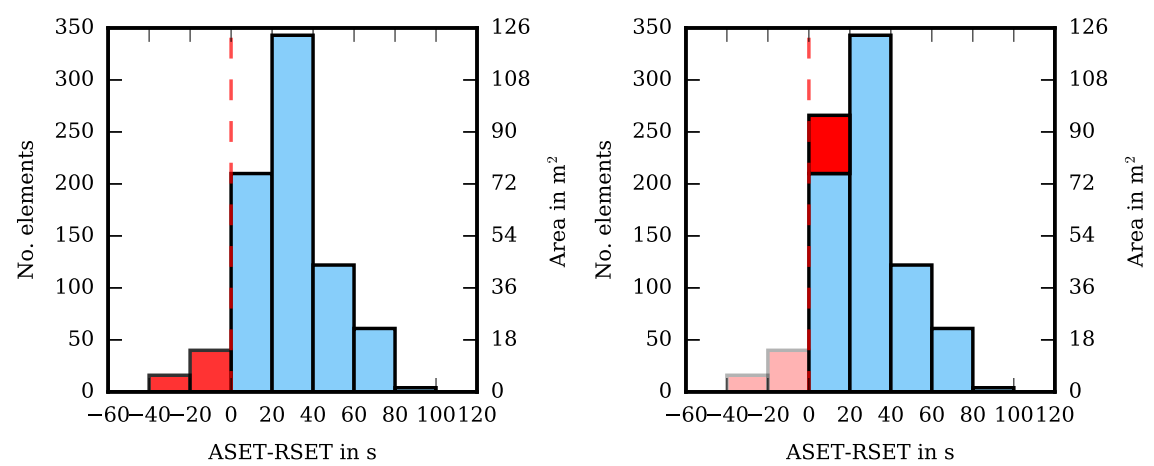

Figure 7: Left column: Histogram derived from the difference map in Figure 4. The limiting state of ASET - RSET $=0$ is marked by a vertical, dashed, red line. The red bins left from that side represent a violation of ASET-RSET. The number of map elements and the corresponding area $A$ in the domain are related by the area of a particular map element (here $A=0.36 \mathrm{~m}^{2}$ ). Right column: Transformation of difference map histogram to the reference state for the EMD calculation. 
On the basis of the histogram, a metric calculation inspired by the so-called "Earth Mover's Distance" (EMD) is introduced. The mathematical basis of EMD is the Wasserstein metric 34, which is used to describe the distance between two distributions. In the proposed method, the first distribution is derived from the difference map of a particular scenario combination. The second distribution is created by the following transformation: the entire negative fraction of the first distribution is shifted back to the limiting state ASET-RSET $=0$. This process can be depicted as moving a particular amount of earth on a certain way back to the limiting state, which explains the naming of this metric.

As stated above, only the bins with negative ASET-RSET values are considered. The norm uses the histogram's bin centres $t_{k}$ and the corresponding accumulated area of the elements $a_{k}$. If the elements have the same area $A$, the accumulated area is given by the product of the number of elements $n_{k}$ and the element area. The result, i.e. the consequences $C$, is the sum of the product of both

$$
C=\sum_{k \mid t_{k}<0} t_{k} \cdot a_{k}=\sum_{k \mid t_{k}<0} t_{k} \cdot n_{k} \cdot A .
$$

It is notable that the original definition of EMD requires normalised distributions. In the context of this work, however, the absolute frequencies of the distributions i.e. areas are incorporated. With regard to the hypothetical scenarios introduced in Figure 6, the measure of consequences $C$ would account to $-19800 \mathrm{~m}^{2} \mathrm{~s}$ for scenario A and to $-32040 \mathrm{~m}^{2} \mathrm{~s}$ for scenario B respectively. Contrary to the temporal and/or spatial extent, $C$ renders scenario B more severe.

Moreover, for more complex built environments, e.g. multi-storey buildings, the consequences of every single difference map per level can be summed up to one total measure of consequences. Regardless of that, the outcome of this method is one scalar measure $C$ which covers the consequences of a scenario combination in terms of ASET-RSET in an extended complexity and robustness. However, the price of these advantages is that the interpretation of this measure is not trivial as it is basically a time weighted sum of areas where the ASETRSET constraint has been violated.

Although the authors do not yet have a direct physical interpretation of $C$, the main objective is to create one scalar measure in order to compare a set of scenario combinations. In other words, there is no primary need that this measure is readily interpretable.

Having said that, the analysis of the parameter study conducted above will be finally extended by the introduced measure of consequences $C$. In the same fashion as for the previous analyses of the spatial and temporal extent, Figure 8 shows the response of $C$ to the parameters number of occupants and maximum heat release rate. For comparison, the temporal extent is provided once again. 


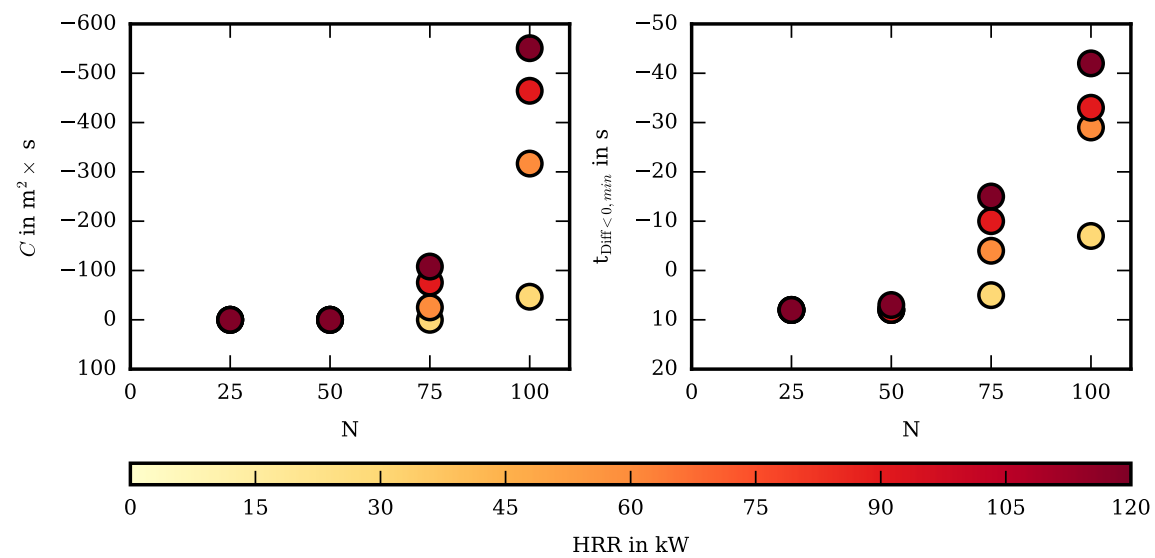

Figure 8: Scatter plots comparing the response of EMD-inspired measure of consequences $C$ (left) compared to the time differences of ASET and RSET (right). Input variations are four different heat release rates and four varying numbers of occupants. The response of both measures reveals similar patterns.

As already concluded in Figure 5. ASET-RSET is increasingly violated in case of a higher number of occupants in conjunction with higher heat release rates. The key finding, however, is that the EMD-inspired measure of consequences $C$ yields a similar pattern as the analysis of the temporal extent does (see Figure 8). The result of this comparative analysis is an indication that $C$ is an appropriate measure to assess a set of scenario combinations regarding ASET-RSET.

But what is the engineering benefit of an additional measure of consequences with limited interpretability? The application of the proposed method to the simplified demonstration case was supposed to demonstrate the appropriateness of the introduced measure. However, its power and the surplus will emerge in scenario combinations with increased complexity. Characteristics of the latter could be multiple fire and evacuation scenarios with in-stationary conditions in multi-storey buildings inducing multiple hot spots where ASET-RSET is violated.

In these cases, the measure $C$ will overcome the limitations of solely evaluating the temporal extent of ASET-RSET, i.e. the safety margin, locally. Instead, the method relives the user from the definition of locations for analysis and the calculated measure $C$ will reliably guide to the most severe scenario combinations regarding the comparison of ASET and RSET. Back to the engineering context, these combinations could than be assessed by further and more detailed analyses.

\section{Limitations}

The limitations of the introduced method are equivalent to the assumptions and shortcomings of the classical ASET-RSET concept itself. From a local per- 
spective, it identifies locations where certain performance criterion has exceeded a predefined threshold while occupants are still present. The approach identifies the limiting state while no further information about the corresponding consequences for individual occupants can be derived. Moreover, the calculated safety margin is conceptually not reversible. For instance, a particular ASET can not be updated when conditions improve, e.g. when activating a venting system. In these cases, it can be beneficial to supplement the analysis by the FED concept.

One benefit of the map implementation is that, in principal, independent fire and evacuation models can be combined during the analysis. However, this may imply limitations on both model levels. For instance, fire dynamics can be influenced by pedestrian behaviour, e.g. opening doors or activating certain fire protection systems manually. In the same way, pedestrian dynamics, e.g. route choice or walking speed, may be affected by the spread of smoke, heat and toxic gases. At least the last-mentioned aspects should play a subordinate role as long as the safety margin is sufficiently greater than the limiting state.

\section{Summary}

In this article, an advancement of the ASET-RSET concept has been introduced. The aim was to adopt the concept to the increased information content of state-of-the-art computer models. With the formal definition of multi-criteria ASET maps and RSET maps, it is possible to calculate difference maps containing a spatial and temporal representation of the safety margin. Moreover, a method is proposed to reduce the complexity of the difference maps to one scalar measure of consequences.

The general formulation is geometry and model independent, i.e. not limited to specific software frameworks or data formats. Additionally, it relieves the user from an a-priori choice of locations that are incorporated in the data analysis. Note that this does not refer to the need that the scenario selection should rely on multiple fire locations. As the analysis is based on areal values, the differences of ASET and RSET can be used to identify critical regions with respect to life safety. The complexity reduction from extensive simulation data over difference maps up to one scalar measure allows to characterise the consequences of a scenario (set) in a more robust way. This facilitates multivariate and quantitative risk analysis concerning ASET-RSET and in turn, counters uncertainties in performance-based design.

Finally, an implementation of the map representation in common simulation tools will allow engineers to use it for daily application.

\section{Acknowledgements}

The idea to conduct life safety analyses with maps arose in the frame of the German research project ORPHEUS (BMBF funding number 13N13266). The focus of this project was fire safety in long-standing underground stations. One of the project's work packages was to conduct multivariate life safety analyses using both fire and evacuation simulation [30]. 
The authors gratefully acknowledge the computing time granted (project jjsc11) by the JARA-HPC Vergabegremium and VSR commission on the supercomputer JURECA [35] at Forschungszentrum Jülich.

\section{References}

\section{References}

[1] National Fire Protection Association, NFPA 101® Life Safety Code® (2018).

[2] DIN 18009-1, DIN 18009-1:2015-04 Brandschutzingenieurwesen - Teil 1: Grundsätze und Regeln für die Anwendung, DIN - Deutsches Institut für Normung (2015).

[3] M. J. Hurley, E. R. Rosenbaum, Performance-Based Design, in: M. Hurley (Ed.), SFPE Handbook of Fire Protection Engineering, 5th Edition, Society of Fire Protection Engineers, 2016, book section 37. doi:10.1007/ 978-1-4939-2565-0.

[4] V. Babrauskas, J. M. Fleming, B. Don Russell, Rset/aset, a flawed concept for fire safety assessment, Fire and Materials 34 (7) (2010) 341-355. arXiv: https://onlinelibrary.wiley.com/doi/pdf/10.1002/fam.1025, doi: 10.1002/fam.1025.

URL https://onlinelibrary.wiley.com/doi/abs/10.1002/fam.1025

[5] R. W. Bukowski, T. E. Waterman, W. J. Christian, Detector sensitivity and siting requirement for dwellings, Tech. Rep. NBS-GCR-75-51, U.S. National Bureau of Standards, Gaithersburg, MD (1975).

[6] L. Y. Cooper, A concept for estimating available safe egress time in fires, Fire Safety Journal 5 (2) (1983) 135 - 144 . doi:https: //doi.org/10.1016/0379-7112(83)90006-1. URL http://www.sciencedirect.com/science/article/pii/ 0379711283900061

[7] S. Gwynnee, K. Boyce, Engineering Data, in: M. Hurley (Ed.), SFPE Handbook of Fire Protection Engineering, 5th Edition, Society of Fire Protection Engineers, 2016, book section 64. doi:10.1007/ 978-1-4939-2565-0.

[8] BS 7974-6, The application of fire safety engineering principles to fire safety design of buildings - Part 6: Human factors: Life safety strategies - Occupant evacuation, behaviour and condition, BSI - British Standards Institution (2004).

[9] ISO/TR 13387, ISO/TR 13387 Fire safety engineering Part 8: Life safety - Occupant behaviour, location and condition, ISO - International Organization for Standardization (1999). 
[10] RiMEA, Guideline for Microscopic Evacuation Analysis, Version 3.0.0, RiMEA e.V. (2016).

[11] G. Heskestad, Fire Plumes, in: M. Hurley (Ed.), SFPE Handbook of Fire Protection Engineering, 2nd Edition, Society of Fire Protection Engineers, 1995, book section 2.1 .

[12] P. Hinkley, Comparison of an established method of calculation of smoke filling of large scale spaces with recent experiments., Fire Science and Technology 8 (1) (1988) 1-8.

[13] E. E. Zukoski, T. Kubota, B. Cetegen, Entrainment in fire plumes, Fire Safety Journal 3 (02 1981). doi:10.1016/0379-7112(81)90037-0.

[14] C. Wade, J. Barnett, A room-corner fire model including fire growth on linings and enclosure smoke-filling, Journal of Fire Protection Engineering 8 (4) (1996) 183-193. doi:10.1177/104239159600800403. URL https://doi .org/10.1177/104239159600800403

[15] G. Walton, W. Dols, CONTAM User Guide and Program Documentation, 2005.

[16] W. W. Jones, R. D. Peacock, G. P. Forney, P. A. Reneke, Cfast - consolidated model of fire growth and smoke transport (version 6), technical reference guide, april 2009 revision, Tech. Rep. Special Publication (NIST SP) - 1026, U.S. National Bureau of Standards, Gaithersburg, MD (2009).

[17] V. M. Predtechenskii, A. I. Milinskii, Planing for foot traffic flow in buildings, Amerind Publishing, New Dehli, 1978, translation of: Proekttirovanie Zhdanii s Uchetom Organizatsii Dvizheniya Lyuddskikh Potokov, Stroiizdat Publishers, Moscow, 1969.

[18] S. M. Gwynne, E. R. Rosenbaum, Employing the Hydraulic Model in Assessing Emergency Movement, in: M. Hurley (Ed.), SFPE Handbook of Fire Protection Engineering, 5th Edition, Society of Fire Protection Engineers, 2016, book section 59. doi:10.1007/978-1-4939-2565-0.

[19] K. McGrattan, S. Hostikka, R. McDermott, J. Floyd, M. Vanella, Fire Dynamics Simulator User's Guide, 6th Edition, National Institute of Standards and Technology, 2019.

[20] J. Ewer, E. Galea, M. Patel, S. Taylor, B. Knight, M. Petridis, Smartfire: an intelligent cfd based fire model, Journal of Fire Protection Engineering 10 (1) (1999) 13-27. doi:10.1177/104239159901000102. URL https://doi.org/10.1177/104239159901000102

[21] ANSYS, Inc., ANSYS CFX Reference Guide, 15th Edition, 2013. 
[22] S. Curtis, A. Best, D. Manocha, Menge: A modular framework for simulating crowd movement, Collective Dynamics 1 (0) (2016) 1-40. doi: 10.17815/CD.2016.1. URL http://collective-dynamics.eu/index.php/cod/article/view/ A1

[23] T. Korhonen, S. Hostikka, S. Heliövaara, H. Ehtamo, Fds+evac: An agent based fire evacuation model, in: W. W. F. Klingsch, C. Rogsch, A. Schadschneider, M. Schreckenberg (Eds.), Pedestrian and Evacuation Dynamics 2008, Springer Berlin Heidelberg, 2010, pp. 109-120. doi: 10.1007/978-3-642-04504-2_8.

URL http://dx.doi.org/10.1007/978-3-642-04504-2_8

[24] Munich University of Applied Sciences - Department of Computer Science and Mathematics, VADERE (2017).

URL http://www . vadere.org/

[25] A. U. Kemloh Wagoum, M. Chraibi, J. Zhang, Jupedsim: An open framework for simulating and analyzing the dynamics of pedestrians, in: 3rd Conference of Transportation Research Group of India, 2015.

[26] E. Galea, J. Perez Galparsoro, A computer-based simulation model for the prediction of evacuation from mass-transport vehicles, Fire Safety Journal 22 (1994) 341-366. doi:10.1016/0379-7112(94)90040-X.

[27] S. Poon, A dynamic approach to aset/rset assessment in performance based design, Procedia Engineering 71 (2014) 173-181.

[28] D. A. Purser, Combustion toxicity, in: M. Hurley (Ed.), SFPE Handbook of Fire Protection Engineering, 5th Edition, Society of Fire Protection Engineers, 2016, book section 62. doi:10.1007/978-1-4939-2565-0.

[29] D. A. Purser, Developments in tenability and escape time assessment for evacuation modelling simulations, in: Evacuation Modeling Trends, Springer, 2016, pp. 25-53.

[30] B. Schröder, Multivariate methods for life safety analysis in case of fire, Ph.D. thesis, Universitätsbibliothek Wuppertal (2017).

[31] B. Schröder, L. Arnold, A. Seyfried, A Map Representation of the ASETRSET Concept (Mar. 2020). doi:10.5281/zenodo.3875550

URL https://doi .org/10.5281/zenodo.3875550

[32] J. Zehfuß, Leitfaden Ingenieurmethoden des Brandschutzes, Report vfdb TB 04-01, Vereinigung zur Förderung des Deutschen Brandschutzes e.V. (2020).

[33] R. Lovreglio, E. Ronchi, D. Borri, The validation of evacuation simulation models through the analysis of behavioural uncertainty, Reliability Engineering \& System Safety 131 (2014) 166-174. doi:http://dx.doi.org/ $10.1016 /$ j.ress.2014.07.007. 
[34] L. N. Vaserstein, Markov processes over denumerable products of spaces, describing large systems of automata, Problems Inform. Transmission 5 (1969) 47-52.

[35] D. Krause, P. Thörnig, JURECA: Modular supercomputer at Jülich Supercomputing Centre, Journal of large-scale research facilities 4 (2018) A132. doi:10.17815/jlsrf-4-121-1.

URL http://juser.fz-juelich.de/record/850758 